\title{
Detección de virus parainfluenza en pacientes inmunocomprometidos e inmunocompetentes atendidos en un hospital terciario de la Ciudad de São Paulo, Brasil
}

\author{
Sheila N. Parmezan, Clarice Camargo, Aripuanã Watanabe, \\ Patricio Godoy-Martínez, Celso Granato y Nancy Bellei
}

\section{Detection of parainfluenza virus in immunocompromised and immunocompetent patients in a tertiary hospital in Sao Paulo, Brazil}

Parainfluenza virus infections (PIV) were evaluated in patients with mild and severe infections through real time PCR. One thousand and sixty-seven samples were collected from subjects as follows: 233 adult renal transplanted outpatients, 129 children with congenital heart disease, 381 with adult hematopoietic stem cell patients and 324 hospitalized patients suspected of influenza A (H1N1) pdm09 infection. PIV was detected in 74 (6.9\%) samples. VPI-3 was the most frequent $(60.8 \%)$ and a higher risk was observed for older adults $(\mathrm{p}=0.018)$ and for those who were hematopoietic stem cell transplanted. Further studies are needed to understand the VPI role in patients' at risk for developing serious illness.

Key words: Parainfluenza virus, real time TR-PCR, viral infection, immunocompromised patients.

Palabras clave: Virus parainfluenza, RPC-TR en tiempo real, infección viral, pacientes inmunocomprometidos.

\section{Introducción}

$\mathrm{E}$ 1 virus parainfluenza (VPI) es un agente prevalente de infecciones respiratorias y se clasifica en cuatro tipos $^{1}$. Este virus es una causa importante de morbilidad en pacientes inmunocomprometidos, pacientes con co-morbilidades previas y en pacientes pediátricos. Los brotes de VPI son comunes en escuelas y unidades de trasplante de precursores hematopoyéticos $(\mathrm{TPH})^{2-4}$.

Después de la aparición de la gripe A (H1N1) pdm09, el número de hospitalizaciones por infecciones respiratorias aumentó drásticamente; los datos indican que otros tipos de virus podrían ser agentes causales de estas complicaciones respiratorias graves en pacientes hospitalizados ${ }^{5,6}$.

Este estudio tiene como objetivo evaluar la detección de los cuatro tipos de VPI en pacientes ambulatorios receptores de trasplantes de riñón, niños con co-morbilidades y adultos hospitalizados con infección respiratoria aguda grave (IRAG), todos ellos atendidos en hospitales terciarios de la ciudad de São Paulo, Brasil.

\section{Pacientes y Métodos}

Este estudio se realizó de forma retrospectiva en el Hospital São Paulo de la Universidad Federal de São Paulo durante los años 2002 a 2012. Se obtuvo un total de 1.067 muestras provenientes de cuatro grupos de pacientes con síntomas respiratorios graves o leves:
- 233 pacientes ambulatorios adultos receptores de trasplante de riñón (TR).

- 129 niños ambulatorios con enfermedad cardíaca congénita (ECC).

- 381 pacientes hospitalizados adultos receptores de trasplante de precursores hematopoyéticos (TPH).

- 324 pacientes hospitalizados con sospecha de gripe A (H1N1) pdm09, en este grupo se dividieron en niños $\leq 12$ años de edad $36 \%(116 / 324)$ y adultos o $>12$ años 53\% (173/324).

Los criterios de inclusión fueron la presencia de probable enfermedad respiratoria de etiología viral, hasta 7 días del inicio de los síntomas y la sospecha de H1N1 grave con diagnóstico clínico de IRAG. Se obtuvo datos demográficos, epidemiológicos y clínicos (tos, coriza, faringodinia/odinofagia, estornudos, mialgia, escalofríos, dolor de cabeza y fiebre). Todos los procedimientos realizados tuvieron la aprobación del Comité de Ética (CEP 0981/2010) del Hospital São Paulo y todos los pacientes dieron su consentimiento informado.

La toma de muestra fue realizada por lavado nasal o hisopado; todas las muestras fueron transportadas al Laboratorio de Virología Clínica y refrigeradas a una temperatura de $4{ }^{\circ} \mathrm{C}$ para el posterior análisis de virus respiratorios. Las muestras fueron procesadas utilizando protocolos publicados anteriormente ${ }^{7}$.

La RT-qPCR (RPC en tiempo real) para los cuatro
Universidad Federal de São Paulo, São Paulo, Brasil. Departamento de Medicina, Unidad de Enfermedades Infecciosas, Laboratorio de Virología Clínica (SNP, CC, AW, CG, NB). Universidad Austral de Chile, Valdivia, Chile.

Facultad de Medicina. Instituto de Microbiología Clínica (PGM)

Los autores no poseen conflictos de interés.

Fuente de Financiamiento: Fundación de Apoyo a la Investigación del Estado de São Paulo (FAPESP), número del protocolo 2010/11888-4.

Recibido: 11 de abril de 2016 Aceptado: 30 de agosto de 2016

Correspondencia a: Nancy Bellei nbellei@uol.com.br 
tipos de VPI se realizó utilizando partidores específicos descritos por van de Pol, 20078; para el VPI-3 se utilizó partidores y sondas específicas para este de acuerdo a Kim, 20119. Las curvas estándares específicas para determinar el límite de detección se llevó a cabo mediante la clonación de los productos de VPI RPC usando kit comercial (TOPO PCR Cloning, Life Technologies, Carlsbad, USA $\left.{ }^{\circledR}\right)$. Los valores de $\mathrm{Ct} £ 37$ para el control endógeno (RNasa P) y $£ 39$ fueron considerados como positivo; este criterio se utilizó para los cuatro tipos de VPI. La técnica para la detección de VPI se realizó en un solo paso, siguiendo el protocolo recomendado para el kit comercial AgPath ID One Step RT-PCR (Life Technologies ${ }^{\circledR}$, Carlsbad, E.U.A.).

Las muestras obtenidas de los pacientes hospitalizados con sospecha de infección por H1N1 se analizaron de acuerdo con el protocolo descrito anteriormente ${ }^{23}$. También fueron analizados otros virus respiratorios: metapneumovirus (MPVh), adenovirus (ADV), y rinovirus $(\mathrm{RVH})^{5}$.

Para el análisis estadístico se utilizó variables categóricas el test de $\chi^{2}$ y el test exacto de Fisher. Se utilizó además la prueba de Levene que es una prueba estadística

Tabla 1. Detección de VPI entre los cuatro grupos de pacientes con síntomas respiratorios graves o leves estudiados ( $n: 1.067$ )

\begin{tabular}{lccccccc}
\hline Grupos* $^{*}$ & $\begin{array}{c}\text { Muestras } \\
(\mathbf{n})\end{array}$ & $\begin{array}{c}\text { Edad } \\
\text { (media) }\end{array}$ & VPI & VPI-1 & $\begin{array}{c}\text { VPI } \mathbf{n} \\
\text { VPI-2 }\end{array}$ & VPI-3 & VPI-4 \\
\hline TR & 233 & 40 & 10 & 2 & 2 & 6 & 0 \\
\hline ECC & 129 & 1 & 9 & 1 & 3 & 3 & 2 \\
\hline sH1N1 & 324 & 15 & 25 & 3 & 5 & 12 & 5 \\
\hline TPH & 381 & 44 & 30 & 1 & 2 & 24 & 3
\end{tabular}

*TR: receptor de trasplante de riñón, adultos, ambulatorio; ECC: niños con enfermedad cardíaca congénita, ambulatorios; sH1N1: pacientes hospitalizados graves con sospecha de infección por A (H1N1) pdm09; TPH: receptor de trasplante de precursores hematopoyéticos. inferencial utilizada para evaluar la igualdad de las varianzas para una variable calculada para dos o más grupos. El programa utilizado para los análisis estadísticos fue el OpenEpi versión 2.3.1. Para todas las pruebas se consideró un valor de $5 \%(\mathrm{p}<0,05)$ como significativo.

\section{Resultados}

El VPI fue detectado durante los dos años que duró el estudio, en especial en otoño. De las 1.067 muestras analizadas, $70 \%(710 / 1015)$ eran adultos y 52,2\% (530) de sexo masculino, la edad media de los adultos fue de 42 años (mediana 41, rango 13-91 años), y la edad media de los niños fue de 3 años (mediana de 1, rango 1-12 años). El VPI se detectó en 6,9\% (74/1067) de las muestras y VPI-3 en 4,2\% (45/1067).

La Tabla 1 muestra la frecuencia de VPI $(\mathrm{p}=0,341)$ y la distribución de edad de los cuatro grupos estudiados ( $p$ $=0,081)$. La comparación de VPI entre los pacientes con TPH y TR arrojó una diferencia estadística significativa de $7,9 \%(30 / 381)$ y $4,3 \%(10 / 233)$, respectivamente ( $p$ $<0,005)$.

La comparación entre los adultos hospitalizados con sospecha de infección por H1N1 (en ausencia de condiciones inmunosupresores conocidas) fue de 4,6\% (7/151) y pacientes con TR o TPH (inmunocomprometidos) de 6,5\% (40/616); no se observó diferencias estadísticamente significativas respecto de las muestras positivas obtenidas para VPI $(p=0,506)$. El análisis de la edad realizado con la prueba de Levene en cada uno de estos grupos mostró una tendencia $(\mathrm{p}=0,068)$, donde el VPI se observó principalmente en el intervalo de edad entre 13-29 años para los individuos inmunocompetentes y el intervalo de 40-60 años para los inmunosuprimidos.

De los 324 pacientes con sospecha de infección por $\mathrm{H} 1 \mathrm{~N} 1,25$ pacientes $(7,7 \%)$ presentaron muestras positivas para el VPI, siendo $18(10,3 \%)$ niños y $7(6,1 \%)$ adultos;
Figura 1. Intervalos de edad en pacientes con los cuatro tipos de virus parainfluenza (VPI).

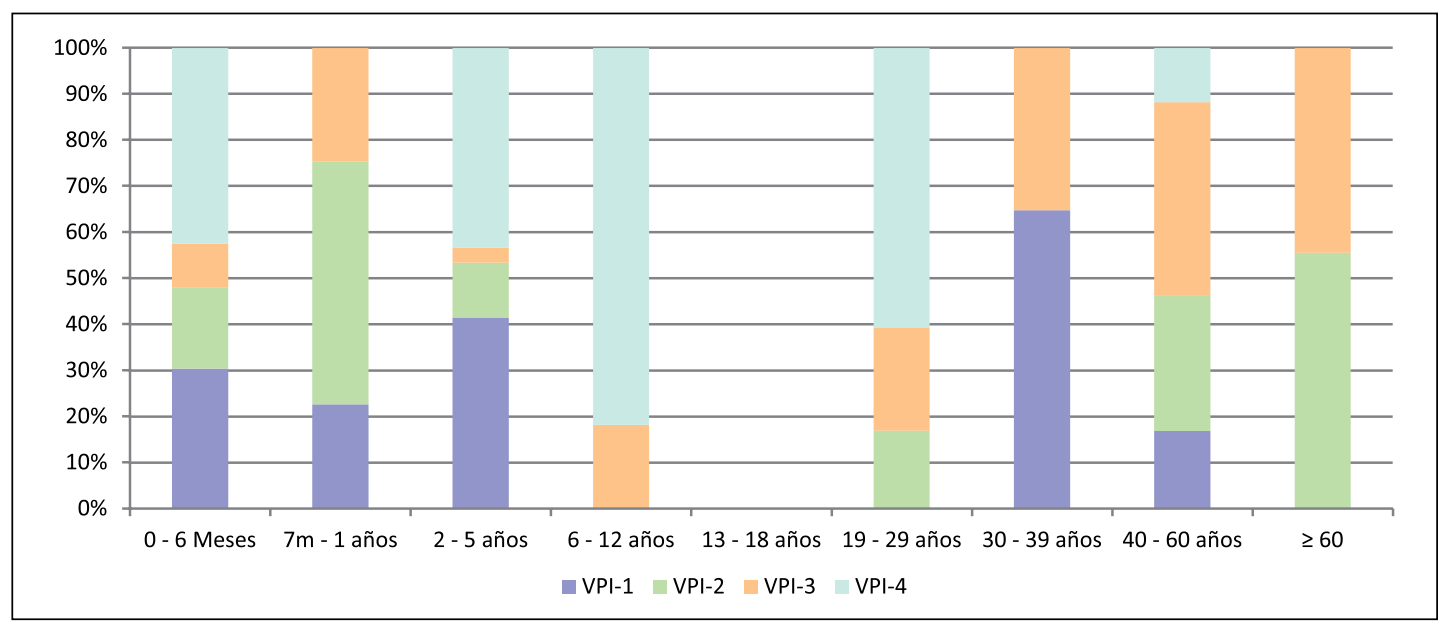


esta diferencia no fue estadísticamente significativa $(p=0,296)$. En este grupo de pacientes el virus influenza (A H1N1 de 2009, H3N2 y B) fue detectado en 23\% de las muestras.

En el análisis de las infecciones por VPI en niños (edad media de tres años, con una mediana de un año) la tasa de positividad fue de $10,4 \%$ (18/173) para niños hospitalizados con sospecha de infección por H1N1 (sin co-morbilidades) y 7,0\% (9/129) para los niños con cardiopatía coronaria $(\mathrm{p}=0,407)$.

VPI-3 fue la infección más detectada, con mayor porcentaje $(72,3 \%)$ en adultos en comparación con los niños $(40,7 \%)(\mathrm{p}=0,056)$. La media y la mediana de edad de VPI-1 fue de 18 y 5 años; para VPI-2 fue de 29 y 15 años; para VPI-3 de 34 y 38 y para VPI-4 de 14 y 5 años.

El análisis de la variación entre los grupos de edad y tipos de VPI mostró una asociación significativa entre el tipo de VPI y la edad $(\mathrm{p}=0,018)$, un mayor porcentaje $(35,6 \%)$ de ocurrencias de se observó para VPI-3 en adultos con edades entre 40 y 60 años en comparación con los otros grupos de edad (Figura 1).

La tasa de detección de más de un virus respiratorio fue de 32,4\% (24/74), siendo rinovirus humano, virus de la gripe estacional humana y adenovirus los que muestran las tasas más altas. No existe asociación entre la detección de los tipos de VPI y la detección concomitante de otros virus respiratorios $(\mathrm{p}=0,071)$.

\section{Discusión}

Después de la pandemia de (H1N1) pdm09, la vigilancia de los casos de síndrome respiratorio aumentó considerablemente, y la demanda de servicios de diagnóstico contribuyó en el aumento de la detección de VPI en pacientes inmunocompetentes e inmunocomprometidos ${ }^{5}$. La tasa de detección de VPI en el presente estudio fue de $6,9 \%$; otros estudios realizados en Brasil mostraron una tasa de positividad de $3,3 \%$ a $10,3 \%{ }^{10,11}$. Estas diferencias observadas en la tasa de detección se debe quizás, al tipo de pacientes (niños hospitalizados o pacientes adultos con trasplante de precursores hematopoyéticos) y las metodologías utilizadas en los otros estudios (inmunofluorescencia y cultivo de células) que poseen una menor sensibilidad en comparación con la RPC en tiempo real ${ }^{12}$.

Se observó que el VPI circula principalmente entre otoño y primavera, con pocos casos detectados en verano. Este patrón también se observó en un estudio realizado en otra ciudad del Estado de São Paulo ${ }^{13}$. A pesar de la mayor circulación en otoño (marzo-junio) y en invierno (junio-septiembre), no se observó un patrón estacional específico lo que podría influir de alguna manera en el diagnóstico. La positividad entre los grupos fue similar, excepto para el grupo de adultos receptores de trasplante de riñón (4,3\%). Estos resultados son discordantes con el estudio de Lo y cols., año $2013^{14}$, que describe a los pacientes adultos con trasplante de precursores hematopoyéticos como el principal grupo de riesgo entre los pacientes inmunocomprometidos.

Puesto que los niños son más vulnerables a las infecciones respiratorias que los adultos sanos ${ }^{15}$, esto podría haber influido en la segunda mayor tasa de positividad 7,7\% (25/324) obtenido para los pacientes hospitalizados con sospecha de gripe A (H1N1) pdm09. Las muestras positivas se detectaron principalmente en los niños $72,0 \%$ (18/25), con una edad promedio de dos años $94,4 \%$ $(17 / 18)$. Otro estudio realizado por Comach y cols., en el año $2012^{16}$ en Venezuela, con pacientes tratados por el síndrome respiratorio agudo grave, encontró que los niños bajo cinco años de edad presentan más positividad para el VPI que niños mayores.

Durante la pandemia y el período inmediato post pandemia de los años 2009 y 2010, se detectó VPI en $7,7 \%$ (25/324) de los pacientes que no presentaron condiciones pre-existentes y que fueron hospitalizados con IRAG. En otros estudios, la frecuencia de los casos graves de VPI varió de 3,8\% (3/79) a 36,0\% (36/100). No se encontró asociación estadística para estos grupos de individuos inmunocompetentes, que tenían entre 13 y 29 años $(\mathrm{p}=0,068)$. Aunque el hecho de que, durante la pandemia, los adultos más jóvenes presentaron mayor riesgo de desarrollar una infección más grave que la gripe A (H1N1) pdm09, una mayor tasa de hospitalización podría contribuir a la comprensión de las otras etiologías ${ }^{6}$.

En el caso de pacientes inmunocomprometidos, no se encontró asociación entre los diferentes grupos de edad $(p=0,068)$, a pesar de la mayor incidencia entre los grupos de 40 a 60 años. Otros estudios mostraron asociación estadística entre este grupo de edad y la infección de virus respiratorios ${ }^{17}$.

El VPI-3 fue el tipo más detectado (aunque no asociado estadísticamente), siendo similar a otros estudios. Una encuesta realizada por el Ministerio de Salud de Brasil con datos de laboratorios de referencia para la vigilancia de los virus respiratorios, confirma los resultados del presente estudio, donde el VPI-3 fue el tipo más común, seguido del VPI-2 y el VPI- ${ }^{18}$. Una posibilidad para explicar la mayor frecuencia de VPI-3 fue la aparición de brotes durante el período del estudio; al respecto hay algunos informes en la literatura médica que describen los brotes en unidades de pacientes inmunocomprometidos ${ }^{19,20}$.

Machado y cols., $2003^{10}$ en Brasil, determinaron una positividad de $10 \%$ de VIP para pacientes receptores de trasplante de precursores hematopoyéticos, utilizando técnicas de inmunofluorescencia directa, pero no detectaron VPI-4. Miall y cols., en el año $2012^{21}$ en Reino Unido, detectaron VPI-4 en un paciente de sexo masculino de 21 años después de un trasplante de precursores hemato- 
poyéticos, diagnóstico que fue confirmado por técnicas moleculares. La detección de VPI-4 en el presente estudio indica la necesidad de aplicar pruebas de diagnóstico más sensibles para VPI-4. La técnica de RPC-TR es importante para la detección de los cuatro tipos de VPI, considerando los estudios que reportan solamente la detección de VPI-1, -2, y -3 ya que utilizan técnicas menos sensibles ${ }^{22}$.

En conclusión, los datos obtenidos en el presente estudio demuestran una mayor tasa de detección de VPI-3 en todos los grupos, especialmente en los adultos mayores hospitalizados y aquellos bajo el programa de trasplante de precursores hematopoyéticos. Actualmente no hay terapias disponibles para el tratamiento de la infección por VPI y la prevención sigue siendo la mejor manera de tratar esta enfermedad. Se necesitan más estudios para comprender el papel de VPI en pacientes de riesgo que pueden desarrollar cuadros clínicos más graves.

\section{Resumen}

Se evaluó la infección por virus parainfluenza (VPI) en pacientes con infecciones leves y graves mediante RPC en tiempo real. Se analizó un total de 1.067 muestras: 233 provenían de pacientes ambulatorios adultos receptores de trasplantes renales, 129 de niños con cardiopatía congénita, 381 de pacientes receptores de trasplantes de precursores hematopoyéticos adultos y 324 de pacientes hospitalizados con sospecha de influenza A (H1N1) pdm09. Se detectó VPI en 74 muestras (6,9\%). Siendo VPI-3 el virus más frecuente $(60,8 \%)$, se observó un mayor riesgo para los adultos mayores $(p=0,018)$ y para aquellos que fueron receptores de precursores hematopoyéticos. Son necesarios estudios adicionales para entender el papel del VPI en pacientes de riesgo para desarrollar enfermedad grave.

\section{Referencias bibliográficas}

1.- Karron R A, Collins P L. 2007. Parainfluenza viruses, p 1502-1526. In: Knipe, D. M.; Howley, P. M, Fields in Virology, 5th ed, New York: Raven Press Ltd.

2.- Henrickson K J. Parainfluenza viruses. Clin Microbiol Rev. 2003; 16 (2): 242-64.

3.- Weinberg G A, Hall C B, Iwane M K, Poehling K A, Edwards K M, Griffin M R, et al. New Vaccine Surveillance Network. Parainfluenza virus infection of young children: estimates of the population-based burden of hospitalization. J Pediatr 2009; 154 (5): 694-9.

4.- Harvala H, Gaunt E, McIntyre C, Roddie H, Labonte S, Curran E, et al. Epidemiology and clinical characteristics of parainfluenza virus 3 outbreak in a Haemato-Oncology unit. J Infect 2012; 65 (3): 246-54.

5.- Watanabe A S, Carraro E, Moreira L, Camargo C, Sinohara J, Puerari D, et al. Respiratory virus infections among hospitalized patients with suspected influenza A H1N1 2009 virus during the first pandemic wave in Brazil. Braz J Infect Dis. 2011; 15 (3): 220-4.

6.- Thiberville S D, Ninove L, Vu Hai V, BotelhoNevers E, Gazin C, Thirion L, et al. The viral etiology of an influenza-like illness during the 2009 pandemic. J Med Virol 2012; 84 (7): 10719.

7.- Bellei N, Carraro E, Perosa A H, Benfica D, Granato C F. Influenza and rhinovirus infections among health-care workers. Respirology 2007; 12 (1): 100-3.

8.- Van de Pol A C, van Loon A M, Wolfs T F, Jansen N J, Nijhuis M, Breteler E K, et al. Increased detection of respiratory syncytial virus, influenza viruses, parainfluenza viruses, and adenoviruses with real-time PCR in samples from patients with respiratory symptoms. J Clin
Microbiol 2007; 45 (7): 2260-2.

9.- Kim C, Ahmed J A, Eidex R B, Nyoka R, Waiboci L W, Erdman D, et al. Comparison of nasopharyngeal and oropharyngeal swabs for the diagnosis of eight respiratory viruses by real-time reverse transcription-PCR assays. PLoS One 2011; 6 (6): e21610.

10.- Machado C M, Boas L S, Mendes A V, Santos M F, da Rocha I F, Sturaro D, et al. Low mortality rates related to respiratory virus infections after bone marrow transplantation. Bone Marrow Transplant 2003; 31 (8): 695-700.

11.- Tsuchiya L R, Costa L M, Raboni S M, Nogueira M B, Pereira L A, Rotta I, et al. Viral respiratory infection in Curitiba, Southern Brazil. J Infect 2005; 51 (5): 401-7.

12.- Kuypers J, Campbell A P, Cent A, Corey L, Boeckh M. Comparison of conventional and molecular detection of respiratory viruses in hematopoietic cell transplant recipients. Transpl Infect Dis 2009; 11 (4): 298-303.

13.- Gardinassi L G, Marques Simas P V, Salomao J B, Durigon E L, Zanetta Trevisan D M, Cordeiro J A, et al. Seasonality of viral respiratory infections in southeast of Brazil: the influence of temperature and air humidity. Braz J Microbiol 2012; 43 (1): 98-108.

14.- Lo M S, Lee G M, Gunawardane N, Burchett S $\mathrm{K}$, Lachenauer C S, Lehmann L E. The impact of RSV, adenovirus, influenza, and parainfluenza infection in pediatric patients receiving stem cell transplant, solid organ transplant, or cancer chemotherapy. Pediatr Transplant 2013; 17 (2): 133-43.

15.- Hsieh Y J, Chin H, Chiu N C, Huang F Y. Hospitalized pediatric parainfluenza virus infections in a medical center. J Microbiol Immunol Infect 2010; 43 (5): 360-5.

16.- Comach G, Teneza-Mora N, Kochel T J, Espino C, Sierra G, Camacho D E, et al. Sentinel surveillance of influenza-like illness in two hospitals in Maracay, Venezuela: 2006-2010. PLoS One 2012; 7 (9): e44511. doi: 10.1371/ journal.pone.0044511.

17.- Chemaly R F, Hanmod S S, Rathod D B, Ghantoji S S, Jiang Y, Doshi A, et al. The characteristics and outcomes of parainfluenza virus infections in 200 patients with leukemia or recipients of hematopoietic stem cell transplantation. Blood 2012; 119 (12): 2738-45.

18.- Freitas F T. Sentinel surveillance of influenza and other respiratory viruses, Brazil, 2000-2010. Braz J Infect Dis 2013; 17 (1): 62-8.

19.- Maziarz R T, Sridharan P, Slater S, Meyers G, Post M, Erdman D D, et al. Control of an outbreak of human parainfluenza virus 3 in hematopoietic stem cell transplant recipients. Biol Blood Marrow Transplant 2010; 16 (2): 192-8.

20.- Sydnor E R, Greer A, Budd A P, Pehar M, Munshaw S, Neofytos D, et al. An outbreak of human parainfluenza virus 3 infection in an outpatient hematopoietic stem cell transplantation clinic. Am J Infect Control 2012; 40 (7): 601-5.

21.- Miall F, Rye A, Fraser M, Hunter A, Snowden J A. Human parainfluenza type 4 infection: a case report highlighting pathogenicity and difficulties in rapid diagnosis in the post-transplant setting. Bone Marrow Transplant 2002; 29 (6): 541-2.

22.- Alonso W J, Laranjeira B J, Pereira S A, Florencio C M, Moreno E C, Miller M A, et al. Comparative dynamics, morbidity and mortality burden of pediatric viral respiratory infections in an equatorial city. Pediatr Infect Dis J 2012; 31 (1): e9-14.

23.- CDC Protocol of real time RT-PCR for influenza A (H1N1). www.who.int/csr/resources/ publications/swineflu/realtimeptpcr/en/index. html 